\title{
ON TWO PROBLEMS OF J. SCHMIDT
}

\section{G. LADNER}

Two problems are raised by J. Schmidt in one of his papers [1]. We shall give solutions, but first introduce certain concepts and definitions on which these problems are based in J. Schmidt's article.

At the outset an arbitrary nonempty set $E$ is fixed.

Denoting the class of all subsets of $E$ by $S(E)$, we shall say that a subclass $K$ of $S(E)$ is a closure class on $E$, if

(i) $E \in K$,

(ii) the intersection $\bigcap_{T \in \mathcal{T}} T$ of every nonempty subclass $\mathcal{T}$ of $K$ is an element of $K$.

Elements of $K$ will be called $K$-closed or, briefly, closed. A closure class $K$ on $E$ is on occasion also denoted by $[E, K]$.

If $K$ is a closure class on $E$, and $M$ an arbitrary subset of $E$, then we mean by the $K$-closure (or, briefly, closure) of $M$ the set

$$
C \mathscr{K}(M)=\bigcap_{M \subseteq K \in \mathscr{K}} K . \quad \text { (Briefly, } C(M) \text { ). }
$$

It follows from the definition of closure class that $C(M)$ is closed.

The function $C=C \mathscr{K}$, defined on $\mathcal{S}(E)$, with values in $\mathcal{S}(E)$, is called a closure operator on $E$. It follows from the definitions that every closure operator $C$ satisfies:

C1. $M_{1} \subseteq M_{2} \rightarrow C\left(M_{1}\right) \subseteq C\left(M_{2}\right)$,

C2. $M \subseteq C(M)$,

C3. $C(C(M))=C(M)$.

Every set-valued set function on $S(E)$ satisfying $C 1, C 2$, and $C 3$ determines a closure class, namely its range, and this correspondence between closure classes on $E$ and closure operators on $E$ is one to one. Two closure operators are called equal, $C_{1}=C_{2}$, if they have the same range.

$\mathcal{T}$ is called a chain in $\mathscr{X} \subseteq \mathcal{S}(E)$ if $\mathcal{T} \subseteq \mathcal{X}$ and if $\mathcal{T}$ is simply ordered by the relation $\subseteq$.

A subclass $\mathcal{X}$ of $\mathcal{S}(E)$ is called inductive if the union of every nonempty chain in $\mathcal{X}$ is an element of $\mathcal{X}$.

A subclass $\mathcal{X}$ of $\mathcal{S}(E)$ is called a hereditary class on $E$, if

$$
M \in \mathcal{X} \text { and } M_{1} \subset M \rightarrow M_{1} \in \mathcal{X} \text {. }
$$

Obviously, $\theta_{E} \in \mathcal{X}$, when $\theta_{E}$ is the empty set and $\mathcal{X}$ a hereditary class on $E$.

Received by the editors September 30, 1954. 
If $K$ is the closure class corresponding to $C=C \mathscr{K}$, then a subset $M$ of $E$ is called $C$-independent (or $K$-independenl), if

$$
M_{1} \subseteq M \text { and } C\left(M_{1}\right)=C(M) \rightarrow M_{1}=M .
$$

Otherwise, $M$ is called $C$-dependent (or $K$-dependent).

It may be easily proved that the class of all independent sets corresponding to a given closure operator $C$ is a hereditary class on $E$. We denote this class by $\Im_{C}$ (or, on occasion, $\Im \mathscr{K}$ ). $\Im_{C}$ is uniquely determined by $C$, but it may happen that

$$
C_{1} \neq C_{2} \text { and } \Im_{C_{1}}=\Im_{C_{2}} \text {. }
$$

(This will be illustrated in the sequel.)

It follows from the definitions and from J. Schmidt's study of inductive closure classes in relation to independence that $J_{C}$ is inductive whenever $C=C \mathscr{K}$ corresponds to an inductive closure class $K$.

It is easy to deduce from relations $\mathrm{C} 1, \mathrm{C} 2$, and $\mathrm{C} 3$ the following

Lemma 1. If $M \subseteq E$ and $x \in C(M)$, then

$$
C(M)=C(\{x\} \cup M) .
$$

This lemma will be used later.

\section{Statement of PRoblems given By J. Schmidt}

QUESTION I. May an arbitrarily given nonempty hereditary class of sets be considered as the class of all $K$-independent sets of a suitable closure class $[E, K]$ ?

Question II. Suppose that $K$ is a closure class such that the class of all $K$-independent sets is inductive. Does this imply that $K$ is inductive?

\section{Solutrons}

I. The answer to question $I$ is in the negative, by virtue of the following

Counterexample. Set $E=E_{6}=\{1,2,3,4,5,6\}$ and consider on $E_{6}$ the hereditary class $\mathfrak{H}$ which consists of all subsets of the sets

$$
P_{1}=\{1,2,3\}, \quad P_{2}=\{3,4,5\}, \quad P_{3}=\{1,5,6\} ;
$$

then there exists no closure operator $C$ on $E_{6}$ satisfying: $\mathfrak{F C}=J_{C}$.

Proof. ${ }^{1}$ Assume: $\exists C$ on $E_{6} \ni \mathcal{H C}=\jmath_{C}$.

Lemma 2. If $\{x, y\} \subset P_{i}(i=1,2$, or 3$)$, then $x \notin C\{y\}$ and $y \notin C\{x\}$.

For by Lemma 1:

1 The following proof is due to W. R. Scott. 


$$
x \in C\{y\} \rightarrow C\{y\}=C\{x, y\},
$$

making $\{x, y\} C$-dependent and contradicting the fact that $C$-independence is a hereditary property. By the same reasoning: $y \notin C\{x\}$, and the lemma is proved.

Consider now the set $\{2,4,6\} \subset E_{6}$. This set, and each of its twoelement subsets, must be $C$-dependent, since they are not contained in any $P_{i}$. Since the two-element subsets as well as the individual elements of $\{2,4,6\}$ possess a cyclic symmetry with respect to $P_{1}, P_{2}$, and $P_{3}$, there results no loss of generality from assuming:

$$
C\{2,4,6\}=C\{2,4\}=C\{2\} \text {. }
$$

Again, there is no loss of generality, if, corresponding to our choice (i), we choose that two-element subset $\{4,6\} \subset\{2,4,6\}$ which does not contain $\{2\}$, and if we assume:

$$
C\{4,6\}=C\{4\} .
$$

Corresponding to the choice (ii), we consider next the set $\{1\}$, which is the intersection of the two sets, $P_{1}$ and $P_{3}$, which do not have (4) as element. From Lemma 2:
(1) $\notin C\{2\}$,
(6) $\notin C\{1\}$.

Now, $\{1,4\}$ must be $C$-dependent. If $C\{1,4\}=C\{4\}$, then (1) $\in C\{4\} \subseteq C\{2,4\}=C\{2\}$. If $C\{1,4\}=C\{1\}$, then $C\{1\} \supseteq C\{4\}$ $=C\{4,6\}$; hence $(6) \in C\{1\}$. Thus, in each of these two cases, a contradiction to Lemma 2 is obtained. From the above-mentioned cyclic symmetry it is obvious that a contradiction of this type will be obtained, regardless of the choice of a two-element set, and of the subsequent choice of a one-element set, by which the required $C$ dependence of the set $\{2,4,6\}$, and of its two-element subsets, may be satisfied. This completes the proof.

II. The answer to question II is in the negative.

Proof. Let $E=R_{1}$ be the set of all real numbers, and consider the closure class $K$ on $R_{1}$ whose elements are all topologically closed symmetric intervals with center (0), where the sets $R_{1}$ and $\{0\}$ are understood to belong to $K$.

If $M \subseteq R_{1}$, then $C \mathscr{K}(M)$ is the least closed interval, centered at (0), which contains $M$. If $M$ has more than one point: suppose $x \in M$, $y \in M,|y| \geqq|x|$. Then obviously $x \in C\{y\}$, hence $C\{x, y\}=C\{y\}$. Thus $\{x, y\}$ is $C$-dependent, and by the heredity property of $\Im_{C}, M$ is $C$-dependent. The set $\{0\}=\bigcap_{\theta_{E} \subset K} \in \mathcal{K} K=C\left(\theta_{E}\right)$ is also $C$-dependent since 


$$
\{0\} \in K \rightarrow C\{0\}=\{0\} \rightarrow C\left(\theta_{E}\right)=C\{0\} .
$$

Every set $\{x\} \ni(0) \neq x \in R_{1}$ is independent by:

$$
C\left(\theta_{E}\right)=\{0\}, \text { and } C\{x\} \neq\{0\} .
$$

Thus the elements of $J_{C}$ are: the empty set $\theta_{E}$ and the sets $\{x\} \ni(0)$ $\neq x \in R_{1}$. The chains in $J_{C}$ are:

(i) the class $\left\{\theta_{E}\right\}$,

(ii) the classes $\{\{x\}\} \ni(0) \neq x \in R_{1}$,

(iii) the classes $\left\{\theta_{E},\{x\}\right\} \ni(0) \neq x \in R_{1}$.

Hence $J_{C}$ is inductive. But $K$ is not inductive, since the union of a chain in $K$ may be an open bounded interval. This completes the proof.

We shall now substantiate an earlier remark, that two different closure operators $C_{1}$ and $C_{2}$ on $E$ may determine the same class of independent sets, $\Im_{C_{1}}=J_{C_{2}}$.

Denote the closure operator on $R_{1}$ corresponding to the closure class used in the preceding proof by $C_{1}$, and the closure operator on $R_{1}$ which corresponds to the closure class $\left\{R_{1},\{0\}\right\}$ by $C_{2}$.

Then obviously: $C_{1} \neq C_{2}$, since the $C_{2}$-closed sets are just the sets $R_{1}$ and $\{0\}$; and clearly $J_{C_{1}}=J_{C_{2}}$.

This example shows also that an inductive closure class may determine the class $\Im \mathcal{K}$ of a noninductive closure class $K$. For the closure class corresponding to $C_{2}$ above is inductive since finite.

\section{BIBLIOGRAPHY}

1. Jürgen Schmidt, Ueber die Rolle der transfiniten Schlussweisen in einer allgemeinen Idealtheorie, Mathematische Nachrichten vol. 7 (1952) pp. 165-182.

UNIVERSITY OF KANSAS 